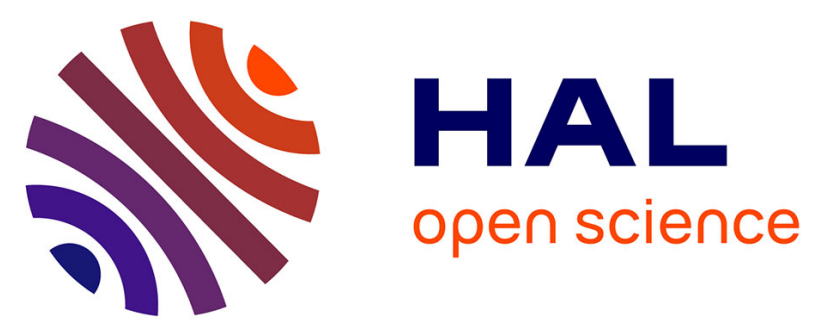

\title{
Evolution en plein champ de la solubilité dans DTPA des métaux lourds du sol introduits par des épandages de boues urbaines chaulées
}

Jean-Louis Morel, Armand Guckert, Anne-Marie Claude, Alain Jeanbille

\section{- To cite this version:}

Jean-Louis Morel, Armand Guckert, Anne-Marie Claude, Alain Jeanbille. Evolution en plein champ de la solubilité dans DTPA des métaux lourds du sol introduits par des épandages de boues urbaines chaulées. Agronomie, 1984, 4 (4), pp.377-386. 10.1051/agro:19840409 . hal-00884648

\section{HAL Id: hal-00884648 \\ https://hal.science/hal-00884648}

Submitted on 1 Jan 1984

HAL is a multi-disciplinary open access archive for the deposit and dissemination of scientific research documents, whether they are published or not. The documents may come from teaching and research institutions in France or abroad, or from public or private research centers.
L'archive ouverte pluridisciplinaire HAL, est destinée au dépôt et à la diffusion de documents scientifiques de niveau recherche, publiés ou non, émanant des établissements d'enseignement et de recherche français ou étrangers, des laboratoires publics ou privés. 


\title{
Evolution en plein champ de la solubilité dans DTPA des métaux lourds du sol introduits par des épandages de boues urbaines chaulées
}

Jean-Louis MOREL \& Armand GUCKERT

avec la collaboration technique de Anne-Marie CLAUde \& Alain JEANBILle

E.N.S.A.I.A., Laboratoire de Phytotechnie, 38, rue Sainte-Catherine, F 54000 Nancy

RÉSUMÉ

\begin{abstract}
A l'aide d'un dispositif expérimental créé en 1974 en vue de déterminer la valeur agronomique de boues urbaines floculées à la chaux et au chlorure ferrique, on a suivi, entre 1979 et 1981, l'évolution de l'extractibilité par DtPa $0,005 \mathrm{M}$ (acide diéthylène triamine penta acétique) de 6 métaux lourds du sol $(\mathrm{Zn}, \mathrm{Cu}, \mathrm{Cd}, \mathrm{Pb}, \mathrm{Ni}$ et Mn). Le plan d'expérience comprend 10 traitements allant de la dose 0 à la dose 340 t M.S. ha ${ }^{-1}$ de boues. Des échantillons de sol ont été constitués pour chaque traitement tous les 6 mois depuis 1979. L'incidence des premiers épandages de boues sur les quantités de métaux extractibles est encore très marquée malgré une interruption des apports pendant 3 ans ; ces quantités sont proportionnelles aux doses de boues épandues. Des épandages récents montrent que les effets d'apports successifs de boues ne sont pas additifs. Au cours du temps, on assiste à la superposition de 2 types de phénomènes antagonistes. Le premier, certainement amplifié par le chaulage des boues, conduit à une réduction globale de l'extractibilité par DTPA ; diverses hypothèses peuvent être formulées pour l'expliquer : diminution des quantités de matières organiques solubles, humification, insolubilisation par précipitation ou adsorption sur les minéraux carbonatés. Le second processus intervenant essentiellement au cours de la saison de végétation entraîne un accroissement de l'extractibilité des métaux lourds par DTPA (biodégradation, acidification, action du système racinaire). Ces augmentations saisonnières s'atténuent cependant très fortement au fur et à mesure que l'on s'éloigne de la date de l'épandage, consécutivement à un probable épuisement des matières organiques labiles et à une organisation des composés minéraux.
\end{abstract}

Mots clés additionnels : $\mathrm{Zn}, \mathrm{Cu}, \mathrm{Cd}, \mathrm{Ni}, \mathrm{Pb}, \mathrm{Mn}$.

DTPA-solubility of soil heavy metals in the field after spreading limed urban sludge.

The mobility of heavy metals in soil after sewage sludge applications was followed in 1979 to 1981 on an experimental field set up in 1974 on a loamy clay soil to evaluate the effects of urban sludges on soil properties and crop production. The various earlier spreadings of sludge resulted in 1979 in 10 experimental treatments (from 0 to $340 \mathrm{t}$ dry matter $\mathrm{ha}^{-1}$ ). For half the treatments, the last sludge application was made in 1975 . Soil samples were collected from the Ap horizon every 6 months. Heavy metal mobility was measured using the DTPA extractant. After 3 years without any application, the soil still showed a strong residual effect of the first sludge spreadings. This was highly correlated with application rate. However, the effects of a new sludge application were not additive. The DTPA-extractable heavy metals decreased with time probably due to the reduction of soluble organic matter, to humification and to mineral insolubilisation, in relation to the limy nature of the sludges. However, after the growing season, heavy metals were shown to be more extractable by DTPA. This was attributed to the action of both microflora and root system (acidification, release of complexing substances). These fluctuations decreased with time.

Additional key words : $Z n, C u, C d, N i, P b, M n$.

\section{INTRODUCTION}

L'épandage agricole constitue une des voies d'utilisation et d'évacuation des boues des stations d'épuration. Cette pratique, intéressant en France $180000 \mathrm{t}$ de matière sèche (Godin, 1982), trouve sa justifica- tion dans la restitution au sol de l'azote, du phosphore et de la matière organique, principaux constituants des boues. En contrepartie, les boues recèlent des quantités notables de substances dont l'introduction au sol n'est pas sans risques pour l'environnement et la santé humaine. Il s'agit de polluants orga- 
niques et d'éléments minéraux parmi lesquels des métaux lourds ( $\mathrm{Zn}, \mathrm{Cu}, \mathrm{Pb}, \mathrm{Ni}, \mathrm{Hg}, \mathrm{Cd}, \ldots)$ sont particulièrement abondants.

La notion de risque pour les métaux lourds est liée, d'une part, à leur toxicité intrinsèque et, d'autre part, à leur solubilité dans le milieu dont dépend leur transfert vers les végétaux. Diverses études de spéciation des métaux lourds (EMMERICH et al., 1982 ; SPOSITO et al., 1982) ont montré que, dans les boues, les formes échangeables et adsorbées sont minoritaires. Selon l'élément, le métal est soit associé à la matière organique $(\mathrm{Cu})$, soit sous forme de carbonates $(\mathrm{Zn}$, $\mathrm{Cd}, \mathrm{Pb}$ ) ou de sulfides $(\mathrm{Ni})$, soit encore sous une forme correspondant à des états très stables chimiquement. La distribution des métaux entre ces diverses formes se trouve aussi étroitement liée à la nature de la boue (SILVIERA \& SOMMERS, 1977) et, en particulier, à la filière de traitement qu'elle a suivie (LINERES \& JUSTE, 1980). Dans le sol, de nombreux processus tels que la biodégradation de la matière organique des boues, l'établissement de liaisons avec les différents constituants et l'existence d'un environnement physico-chimique différent $(\mathrm{pH}, \mathrm{Eh})$ contribuent à modifier l'état des métaux lourds introduits par la boue. Selon les conditions, ces modifications d'état interviennent de différentes manières : c'est ainsi que SILVIERA \& SOMMERS (1977) observent au cours d'incubations de mélanges sol + boues, une augmentation de la disponibilité des métaux au cours du temps alors que, de leur côté, FolLET \& LINDSAY (1971), CHANG \& BROADBENT (1982), avec des métaux introduits sous forme de sels, assistent à la diminution de leurs possibilités d'extraction par DTPA. A plus long terme, il semblerait que les métaux, consécutivement à leur introduction dans le sol, évoluent vers des formes chimiques plus stables (EMMERICH et al., 1982) beaucoup moins assimilables par les végétaux. Ce phénomène, équivalent de la « reversion » connue en fertilisation oligominérale, a pu être observé à l'occasion de cultures, en présence de boues, qui montraient une diminution du prélèvement des métaux lourds par les végétaux à mesure çue l'on s'éloignait de la date de l'épandage (JUSTE \& SOLDA, 1979 ; GUCKERT et al., 1982).

En vue de préciser le devenir dans le sol de métaux lourds apportés par les boues, nous avons suivi pendant 3 années à l'aide d'un extractant chimique, le DTPA (acide diéthylène triamine penta acétique), l'évolution de la fraction présumée assimilable de $\mathrm{Zn}$, $\mathrm{Cu}, \mathrm{Cd}, \mathrm{Pb}, \mathrm{Ni}$ et $\mathrm{Mn}$ dans un sol enrichi de différentes doses de boues urbaines floculées à la chaux et au chlorure ferrique. Ce type de traitement, assez répandu, confère aux boues des propriétés agronomiques particulières : évolution de leur matière organique (MOREL \& GUCKERT, 1981), aptitude à fournir l'azote (CHAUSSOD, 1979) et le phosphore (FARDEAU et al., 1977 ; MOREL, 1980), comportement des métaux lourds (FARDEAU et al., 1977 ; JUSTE et al., 1977).

\section{MATÉRIEL ET MÉTHODES}

\section{A. Sol}

Le sol, de type brun lessivé, développé sur une couverture de limons quaternaires $(30$ à $40 \mathrm{~cm})$, repose sur un substrat marneux au voisinage duquel apparaît un horizon de pseudogley. Le tableau 1 donne les caractéristiques analytiques de l'horizon de surface de ce sol.

\section{B. Boues}

Elles proviennent de la station d'épuration biologique de la communauté urbaine de Nancy qui produit quotidiennement environ $60 \mathrm{t}$ de boues à $20 \mathrm{p} .100 \mathrm{de}$ matière sèche. Avant d'être évacuées, les boues sont digérées en anaérobiose, puis floculées à la chaux $\left(20\right.$ p. $100 \mathrm{Ca}(\mathrm{OH})_{2}$ par rapport à la matière sèche) et au chlorure ferrique, enfin déshydratées sur filtre rotatif sous vide. Ce mode de conditionnement confère à la boue un caractère d'amendement calcaire susceptible d'induire un comportement particulier des métaux dans le sol. Une analyse moyenne des boues par rapport à la matière sèche est rapportée tableau 1, les variations dans le temps de la composition pouvant être importantes (MOREL et al., 1978).

\section{Dispositif expérimental}

Installé en 1974 sur le domaine expérimental de la Bouzule (Meurthe-et-Moselle) pour suivre les répercussions agronomiques de l'épandage des boues de Nancy, il présentait initialement 5 traitements à doses croissantes de boues (tabl. 1), la moitié du dispositif recevant un complément d'engrais minéral. A partir de mars 1979, date du début de la présente étude, les épandages de boues ont été renouvelés uniquement sur la moitié des parcelles. Il en est résulté un dispositif comportant 10 traitements allant de la dose zéro à la dose $340 \mathrm{t}$ M.S. ha ${ }^{-1}$ en quantité cumulée : 4 traitements représentant l'arrière effet des boues (pas de nouvel apport, mais application de $120 \mathrm{~kg} \cdot \mathrm{ha}^{-1} \mathrm{~N}$, $\left.\mathrm{P}_{2} \mathrm{O}_{5}, \mathrm{~K}_{2} \mathrm{O}\right), 4$ traitements correspondant aux épandages de 1979,1 traitement témoin $O$ boue et 1 traitement témoin $+120 \mathrm{~kg} . \mathrm{ha}^{-1} \mathrm{~N}, \mathrm{P}_{2} \mathrm{O}_{5}, \mathrm{~K}_{2} \mathrm{O}$. Chaque traitement présente 4 répétitions sous forme de parcelles de $10 \mathrm{~m}^{2}$. L'étude de l'évolution des teneurs du sol en métaux lourds assimilables a été envisagée après le dernier apport de boues (1979), soit 5 ans après son installation, période qui garantit au mélange sol + boue un certain état d'équilibre. Durant les 3 années de cette étude, les parcelles ont été cultivées en maïs ensilage (var. « L.G. $11 »)$. A la récolte, les parties aériennes (tige, feuilles, épis) ont été exportées, seules ont été restituées la base de la tige et les racines, enfouies par un labour à $20 \mathrm{~cm}$ de profondeur. Les sols ont été prélevés 2 fois par an dans l'horizon de surface, avant et après la culture, soit en mars et en octobre. Chaque échantillon constitué à partir de 10 prélèvements à l'aide d'une sonde cylindrique $(\varnothing: 1 \mathrm{~cm})$ a été émietté, puis séché à l'air libre, ensuite pulvérisé dans un mortier en porcelaine et tamisé à $2 \mathrm{~mm}$ avant d'être analysé.

\section{Analyses}

\section{Métaux totaux}

Minéralisation à chaud de $2 \mathrm{~g}$ de sol dans $20 \mathrm{ml}$ de solution nitro-perchlorique. Dosage de $\mathrm{Zn}, \mathrm{Cu}, \mathrm{Cd}$, $\mathrm{Pb}, \mathrm{Ni}, \mathrm{Mn}$ dans la solution par absorption atomique (Varian AA6). 
TABLEAU 1

Analyse* de l'horizon Ap du sol (sol témoin prélevé en mars 1979) composition moyenne* des boues et principales caractéristiques du dispositif expérimental.

Soil analysis (check soil in March 1979), average sludge composition and basic experimental lay-out.

Sol

\begin{tabular}{|c|c|c|c|c|c|c|c|}
\hline \multirow{2}{*}{$\mathrm{pH}$} & \multicolumn{5}{|c|}{ Granulométrie } & \multirow{2}{*}{$\mathrm{C}$} & \multirow{2}{*}{$\mathrm{CEC}$} \\
\hline & $\begin{array}{c}\dot{A} \\
<2 \mu\end{array}$ & $\begin{array}{c}\text { LF } \\
2-20 \mu\end{array}$ & $\begin{array}{c}\text { LG } \\
20-50 \mu\end{array}$ & $\underset{50-200 \mu}{\text { SF }}$ & $\begin{array}{c}\text { SG } \\
200-2000 \mu\end{array}$ & & \\
\hline & \multicolumn{5}{|c|}{$\%$} & & \multirow{2}{*}{$\begin{array}{c}\mathrm{me} / 100 \mathrm{~g} \\
16,6\end{array}$} \\
\hline 7,1 & 29,3 & 36,0 & 19,0 & 6,2 & 8,5 & 1,76 & \\
\hline
\end{tabular}

Boues

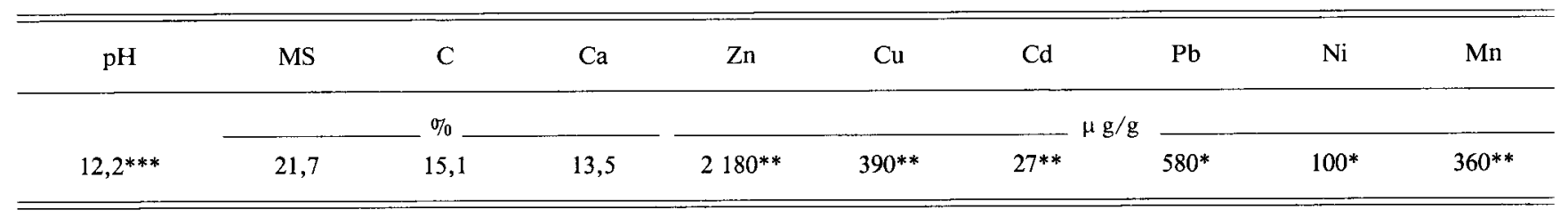

Dispositif expérimental

\begin{tabular}{|c|c|c|c|c|c|c|c|c|}
\hline $\begin{array}{l}\text { Epandages de } \\
\text { boues en } 1974 \\
\text { et } 1975\end{array}$ & $\begin{array}{l}\text { Epandages de } \\
\text { boues floculées } \\
\text { en } 1979\end{array}$ & $\begin{array}{c}\text { Quantités totales } \\
\text { de boues épandues } \\
1974-1981\end{array}$ & \multicolumn{6}{|c|}{$\begin{array}{c}\text { Quantités totales de métaux } \\
\text { lourds appliquées } \\
1974-1981\end{array}$} \\
\hline & t M.S. ha ${ }^{-1}$ & & \multicolumn{6}{|c|}{$\mathrm{kg} \cdot \mathrm{ha}^{-1}$} \\
\hline 0 & $\begin{array}{l}\mathrm{E} \\
0\end{array}$ & $\begin{array}{l}0 \mathrm{e} \\
0\end{array}$ & $\mathrm{Zn}$ & $\mathrm{Cu}$ & $\mathrm{Cd}$ & $\mathrm{Pb}$ & $\mathrm{Ni}$ & $\mathrm{Mn}$ \\
\hline $30^{\bullet}$ & $\begin{array}{c}\mathrm{E} \\
10\end{array}$ & $\begin{array}{l}30 \\
40\end{array}$ & $\begin{array}{l}65 \\
87\end{array}$ & $\begin{array}{l}11,7 \\
15,6\end{array}$ & $\begin{array}{l}0,81 \\
1,08\end{array}$ & $\begin{array}{l}17,4 \\
23,2\end{array}$ & $\begin{array}{l}3,3 \\
4,4\end{array}$ & $\begin{array}{l}10,8 \\
14,4\end{array}$ \\
\hline 70 & $\begin{array}{c}\mathrm{E} \\
30\end{array}$ & $\begin{array}{r}70 \\
100\end{array}$ & $\begin{array}{l}153 \\
218\end{array}$ & $\begin{array}{l}27,3 \\
39,0\end{array}$ & $\begin{array}{l}1,89 \\
2,70\end{array}$ & $\begin{array}{l}40,6 \\
58,0\end{array}$ & $\begin{array}{r}7,7 \\
11,0\end{array}$ & $\begin{array}{l}25,2 \\
36,0\end{array}$ \\
\hline 140 & $\begin{array}{l}\mathrm{E} \\
60\end{array}$ & $\begin{array}{l}140 \\
200\end{array}$ & $\begin{array}{l}305 \\
436\end{array}$ & $\begin{array}{l}54,6 \\
78,0\end{array}$ & $\begin{array}{l}3,78 \\
5,40\end{array}$ & $\begin{array}{r}81,2 \\
116,0\end{array}$ & $\begin{array}{l}15,4 \\
22,0\end{array}$ & $\begin{array}{l}50,4 \\
72,0\end{array}$ \\
\hline $190^{\bullet}$ & $\begin{array}{c}\mathrm{E} \\
150\end{array}$ & $\begin{array}{l}190 \\
340\end{array}$ & $\begin{array}{l}414 \\
741\end{array}$ & $\begin{array}{r}74,1 \\
132,6\end{array}$ & $\begin{array}{l}5,13 \\
9,18\end{array}$ & $\begin{array}{l}110,2 \\
197,2\end{array}$ & $\begin{array}{l}20,9 \\
37,4\end{array}$ & $\begin{array}{r}68,4 \\
122,4\end{array}$ \\
\hline \multicolumn{4}{|c|}{$\begin{array}{l}\text { * Par rapport à la matière sèche. } \\
\text { ** Moyenne de l'analyse de } 19 \text { échantillons. } \\
{ }^{* * *} \text { pH } 9 \text { après séchage de la boue. } \\
30^{\bullet}: 30 \text { t M.S. ha }{ }^{-1} \text { boues non floculées. } \\
190^{\bullet}: 60 \text { t M.S. ha }{ }^{-1} \text { boues non floculées }+130 \text { t M.S. ha }{ }^{-1} \text { boues floculées. } \\
\text { E : Apport de } 120 \mathrm{~kg}^{-} \text {ha }{ }^{-1} \text { d'engrais } \mathrm{N}, \mathrm{P}_{2} \mathrm{O}_{5}, \mathrm{~K}_{2} \mathrm{O} \text {. }\end{array}$} & \multicolumn{5}{|c|}{ 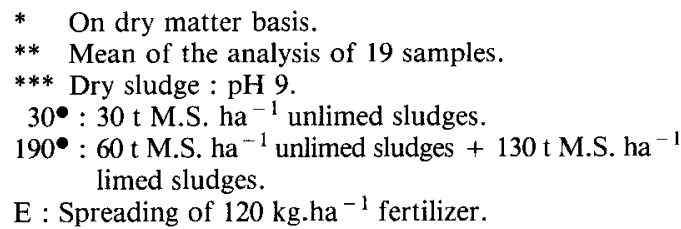 } \\
\hline
\end{tabular}

\section{Métaux assimilables}

La fraction des métaux lourds disponible pour les végétaux peut être appréciée avec plus ou moins de succès à l'aide de nombreuses méthodes chimiques. Celles-ci diffèrent essentiellement par le type d'agent d'extraction, qui peut être un acide $\left(\mathrm{HCl}, \mathrm{HNO}_{3}\right.$, $\left.\mathrm{CH}_{3} \mathrm{COOH}\right)$, un sel $\left(\mathrm{NH}_{4} \mathrm{CH}_{3} \mathrm{COO}, \mathrm{CaCl}_{2}\right)$ ou un chélate (EDTA, DTPA, NTA). Ce mode d'approche est critiquable dans la mesure où l'on n'est pas assuré d'obtenir, selon les conditions de milieu, une image représentative du pool des métaux assimilables. Par contre, la technique d'extraction chimique représente un moyen pratique et intéressant pour comparer des niveaux de contamination différents entre eux et pour suivre leur évolution. $\mathrm{Si}$, en France, il semble que l'on s'oriente vers l'utilisation de l'EDTA (JUSTE, 1983), un autre agent complexant, le DTPA, massivement employé dans le cadre de l'analyse des éléments traces assimilables dans les sols (LINDSAY \& NoRVELL, 1978) et de l'étude de la contamination des sols par les métaux lourds (KELLING et al., 1977 ; KORCAK \& FANNING, 1978 ; LATTEREL et al., 1978 ; HAQ et al., 1980 ; LINERES \& JUSTE, 1980), autorise ce type de travail comparatif. Le DTPA permet d'accéder aux ions métal libres et facilement désorbables du sol. Nous avons employé la méthode de LINDSAY \& NORVELL (1978) : agitation, pendant $2 \mathrm{~h}$, de $10 \mathrm{~g}$ de sol dans une solution de DTPA $0,005 \mathrm{M}, \mathrm{CaCl}_{2} 0,1 \mathrm{M}$ et de TEA (triéthanolamine) $0,1 \mathrm{M}$ tamponnée à $\mathrm{pH}$ 7,3 ; après filtration, analyse de la solution par absorption atomique pour $\mathrm{Zn}, \mathrm{Cu}, \mathrm{Cd}, \mathrm{Pb}, \mathrm{Ni}$ et $\mathrm{Mn}$. 


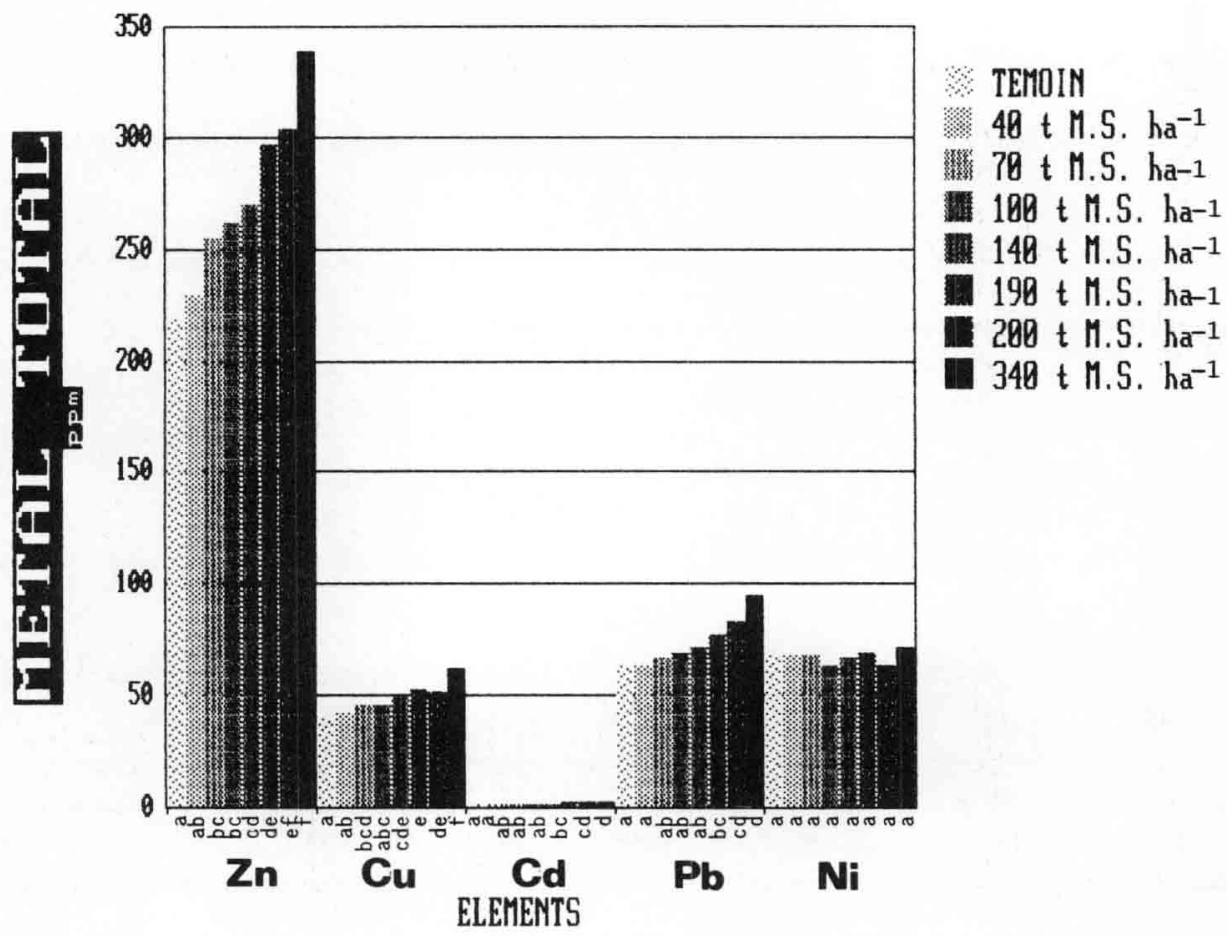

Figure 1

Teneur du sol en métaux lourds à la fin de l'expérimentation. Notes :

- corrélations dose-concentration : $\mathrm{Zn}: r=0,99 ; \mathrm{Cu}: r=0,99$; $C d: r=0,94, P b: r=0,98$;

- pour un élément, les barres affectées de la même lettre ne sont pas significativement différentes au niveau $5 \%$ (test de DuN. CAN);

- témoin; moyenne de 8 répétitions (témoin 0 et témoin 0e);

- traitement 30 t M.S. ha ${ }^{-1}$ et manganèse non représentés.

\section{RÉSULTATS}

Avant de décrire l'évolution au cours du temps de la solubilité dans le DTPA des métaux lourds apportés par les boues, nous examinerons les conséquences de 8 années d'utilisation de boues urbaines sur la teneur totale du sol en métaux lourds.

\section{A. Concentrations du sol en métaux lourds totaux après 8 années d'épandage des boues urbaines}

La figure 1 indique les concentrations totales des éléments étudiés, à la fin 1981. L'application des boues s'accompagne d'un accroissement significatif de la teneur du sol en $\mathrm{Zn}, \mathrm{Cu}, \mathrm{Cd}$ et $\mathrm{Pb}$ totaux. Par contre, on n'observe aucune modification des teneurs en $\mathrm{Mn}$ et Ni totaux, eu égard au faible rapport : concentration du métal dans la boue/concentration initiale dans le sol, présenté par ces 2 éléments. Il existe une étroite proportionnalité entre la concentration totale de $\mathrm{Zn}, \mathrm{Cu}, \mathrm{Cd}$ et $\mathrm{Pb}$ retrouvée dans le sol et la quantité totale de boue épandue depuis l'implantation du dispositif (coefficients de corrélation: $0,94<\mathrm{r}<0,99$ ). La prévision de la concentration du sol en métal total, faite à partir de la concentration de la boue et de la dose appliquée, aboutit à des valeurs largement plus élevées que celles effectivement données par l'analyse de sol (on ne retrouve par l'analyse que 50 p. 100 environ de la concentration
Total heavy metal concentration in the Ap soil horizon at the end of the experiment.

Notes:

- bars preceded by the same letter do not differ at the 0.05 level (DUNCAN test):

- the sludge rate/metal concentration correlations were $\mathrm{Zn}$ : $r=0.99 ; C u: r=0.99 ; C d: r=0.94 ; P b: r=0.98$;

- check: mean of 8 replicates (0 and De treatments). 30 t D.M. ha $a^{-1}$ and manganese are not represented.

estimée). Une telle divergence a été également observée par ROBERTSON et al. (1982) et imputée, en partie, à des erreurs d'échantillonnage et d'analyse. Si ces causes d'erreurs sont vraisemblables, en particulier pour les boues dont la composition est sujette à d'importantes variations dans le temps (MOREL et al., 1978), le mode d'expression des résultats est aussi conditionné par la profondeur de labour et la densité apparente du sol retenues.

\section{B. Evolution des teneurs du sol en métaux extracti- bles au DTPA}

Après avoir décrit pour chaque métal l'état du dispositif 5 ans après son implantation (mars 1979), nous opposerons les parcelles sans apport de boues depuis 1975 et ayant atteint un certain état d'équilibre à celles enrichies en boues en mars 1979.

\section{Zinc (fig. 2)}

En mars 1979, la quantité de $\mathrm{Zn}$ extractible au DTPA ( $\mathrm{Zn}$-DTPA) est étroitement liée au $\mathrm{Zn}$ apporté par les épandages de boues antérieurs $(r=0,95)$. De $3,4 \mathrm{ppm}$ dans le sol témoin, niveau qui selon LINDSAY \& NORVELL (1978) correspond à un sol correctement pourvu, cette quantité est passée à $26,5 \mathrm{ppm}$ avec le traitement $340 \mathrm{t}$ M.S. ha- ${ }^{-1}$. Remarquons qu'un traitement $30 \mathrm{t}$ M.S. ha ${ }^{-1}$ de boues non chau- 


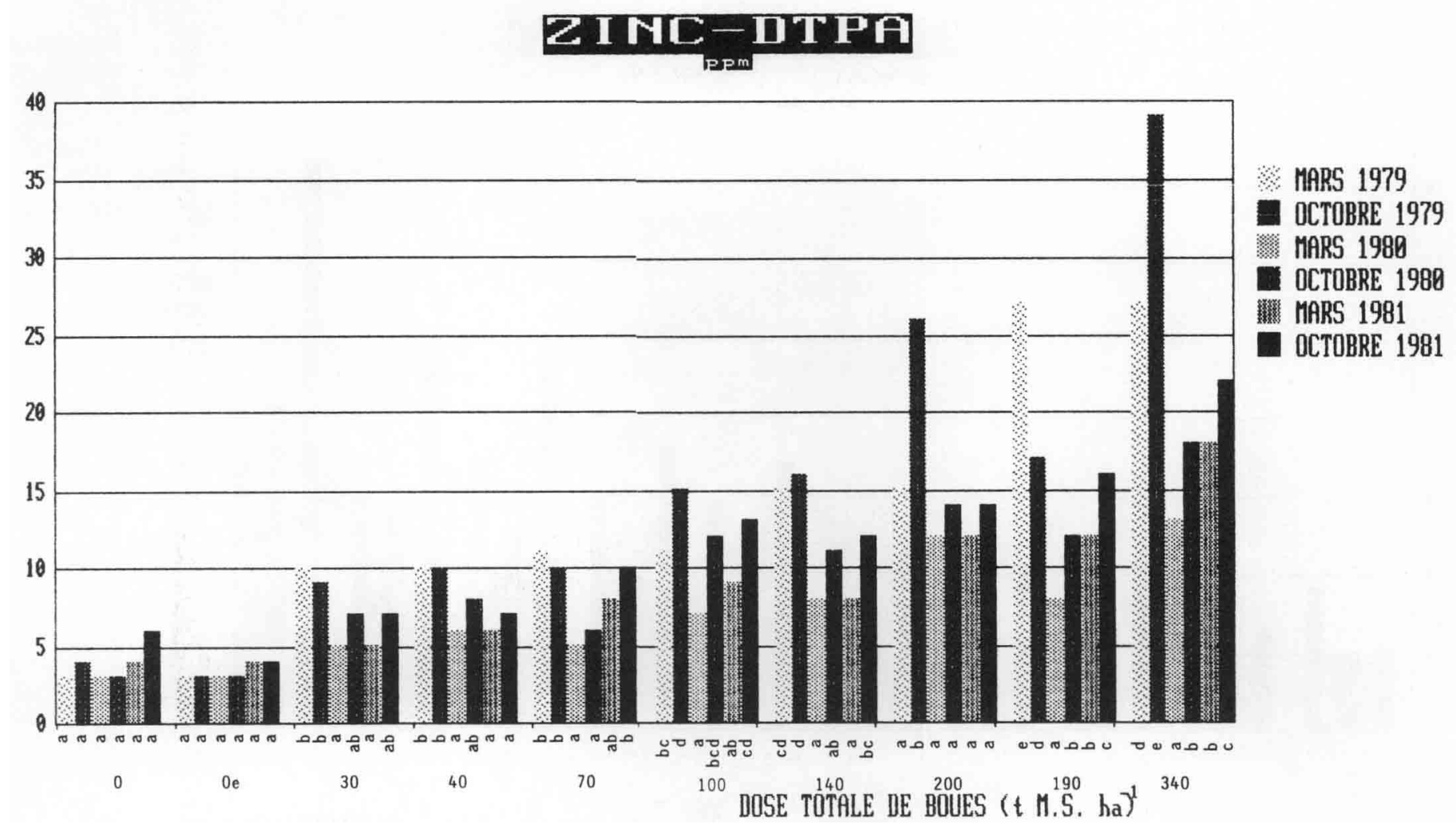

Figure 2.

Evolution de la teneur du sol en Zn extractible par DTPA pour les différents traitements boues.

Notes :

- En mars 1979, les traitements De, 30, 70, 140 et $190 \mathrm{t}$ M.S. ha $\mathrm{a}^{-1}$ ont recu $120 \mathrm{~kg} . \mathrm{ha}^{-1} \mathrm{~N}, \mathrm{P}_{2} \mathrm{O}_{9}, \mathrm{~K}_{2} \mathrm{O}$ et les traitements 40,100 , 200 et $340 \mathrm{t} M . S$. / ha respectivement $10,30,60$ et $150 \mathrm{tM}$.S. $\mathrm{ha}^{-1}$ de boues chaulées.

- Les prélèvements de sol de mars 1979 ont été réalisés avant les nouveaux épandages.

- Pour un même traitement, les barres affectées de la même lettre ne sont pas significativement différentes au niveau 5 p. 100 (test de DUNCAN).

Change in soil DTPA-extractable $\mathrm{Zn}$ for the different treatments.

Notes :

- In March 1979, application of $120 \mathrm{~kg} \cdot \mathrm{ha}^{-1} \mathrm{~N}, \mathrm{P}_{2} \mathrm{O}_{5}, \mathrm{~K}_{2} \mathrm{O}$ as soluble fertilizer for $0 e, 30,70,140$ and $190 \mathrm{t} \mathrm{D.M.} \mathrm{ha} \mathrm{I}^{-I}$ and of 10, 30, 60 and 150 t D.M. ha $a^{-1}$ of flocculated sludges for 0 ,

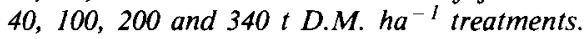

- 1979 soil sampling done before sludge spreading.

- For a treatment, bars preceded by the same letter do not differ at the 0.05 level (DUNCAN test).

lées a le même effet sur la teneur du sol en Zn-DTPA qu'un traitement 70 t M.S. ha ${ }^{-1}$ de boues chaulées. Sans nouvel apport de boues, les niveaux de $\mathrm{Zn}$ DTPA tendent à diminuer au cours du temps. En mars 1980 , ils s'abaissent systématiquement à moins de $10 \mathrm{ppm}$. Toutefois, en octobre, la concentration du sol en Zn-DTPA montre une augmentation que nous qualifierons de saisonnière. Sur les parcelles enrichies en boues en 1979, on observe, après la récolte de l'année, un accroissement de la concentration en $\mathrm{Zn}$-DTPA, exception faite de la dose d'apport la plus faible $(10 \mathrm{t} \mathrm{M.S.} \mathrm{ha-1)}$. Les valeurs atteignent $40 \mathrm{ppm}$ avec un épandage de $150 \mathrm{t}$ M.S. ha- ${ }^{-1}$. Compte tenu des quantités de boues appliquées en 1979 , on aurait pu s'attendre à une augmentation plus importante des concentrations du sol en $\mathrm{Zn}$ extractible. En mars de l'année suivante, les niveaux baissent de manière très spectaculaire et atteignent des valeurs toutes inférieures à $15 \mathrm{ppm}$. De même que précédemment, on assiste à des fluctuations entre mars et octobre.

\section{Cuivre (fig. 3)}

Comme pour $\mathrm{Zn}$, les valeurs initiales de Cu-DTPA croissent linéairement avec les apports de cuivre par les boues. La corrélation est ici meilleure $(r=0,99)$, le traitement $30 \mathrm{t} \mathrm{M.S.} \mathrm{ha}{ }^{-1}$ ne faisant pas exception. Avec 140 et 190 t M.S. ha-1, les quantités de $\mathrm{Cu}$ extractible par DTPA diminuent régulièrement au cours du temps et montrent aussi une légère remontée après chaque récolte. Pour les autres traitements, les variations sont peu marquées d'une saison à l'autre. L'épandage de 1979 a produit une large augmentation de Cu-DTPA en octobre 1979, hormis pour le traitement $10 \mathrm{t}$ M.S. ha ${ }^{-1}$. En mars 1980 , on assiste à une nette réduction de Cu-DTPA, qui atteint le niveau des parcelles sans boues en 1979 correspondantes. Toutefois, en octobre de l'année suivante, il se produit une remontée importante créant à nouveau des différences entre ces 2 types de traitements.

\section{Cadmium (fig. 4)}

Le comportement du cadmium extractible au DTPA est similaire à celui du zinc. La quantité de Cd-DTPA est liée linéairement à la dose totale de $\mathrm{Cd}$ appliquée $(r=0,96)$. Cependant, malgré l'existence de variations saisonnières, la diminution au cours du temps est plus régulière et plus forte. On note également que la dose 190 t M.S. ha ${ }^{-1}$ offre des valeurs systématiquement supérieures à celles de la dose $200 \mathrm{t}$ 


\section{CUIURE-DTPA}

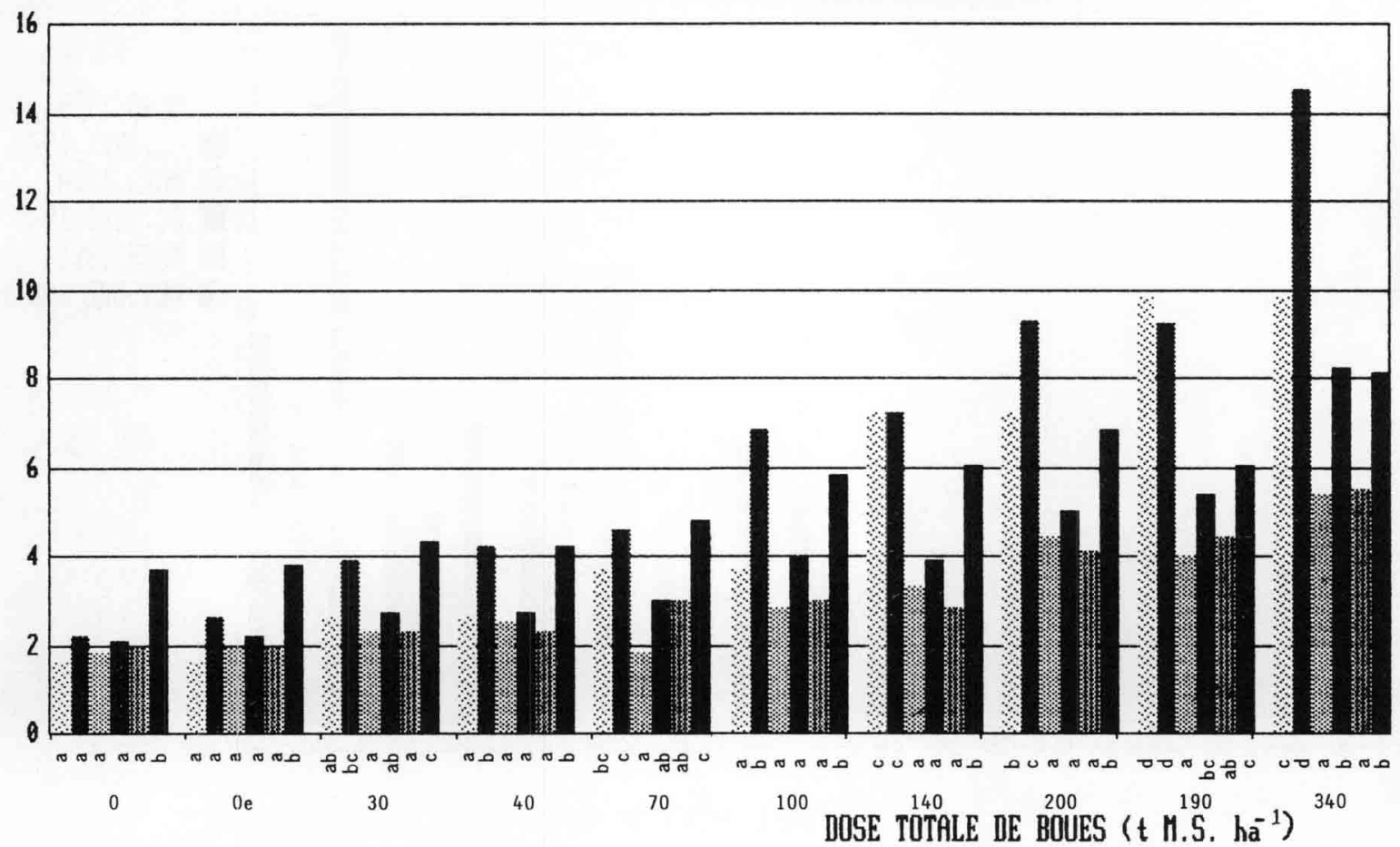

MARS 1979

OCTOBRE 1979

MARS 1980

ECTOBRE 1980

醀 MARS 1981

OCTOBRE 1981

Figure 3

Evolution de la teneur du sol en Cu extractible par le DTPA pour les différents traitements boues. Notes : Voir figure 2.
Change in soil DTPA extractable Cu for the different treatments. Notes : see figure 2.

\section{CADTI पT-DTPA}

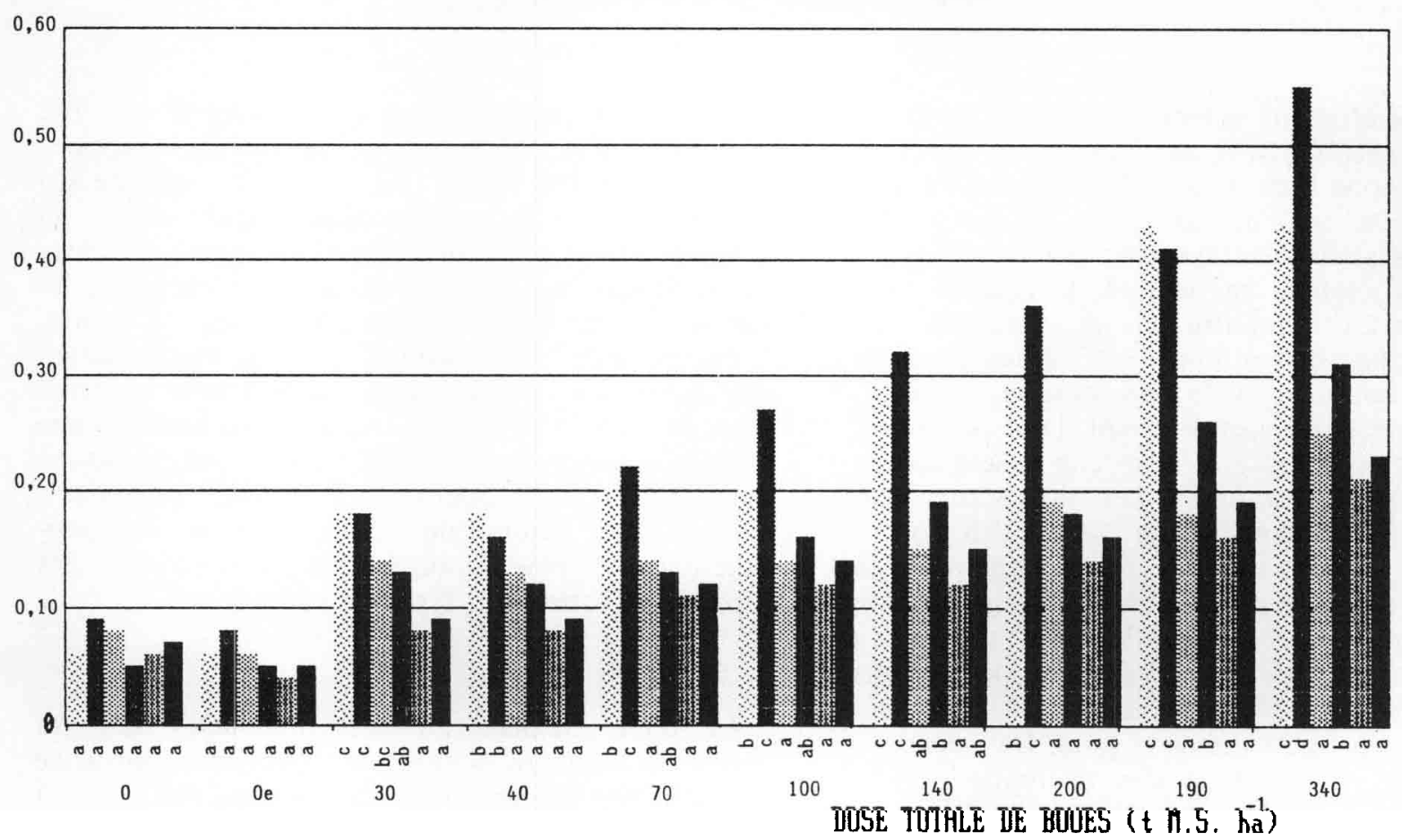

MARS 1979

OCTOBRE 1979

MARS 1989

OCTOBRE 1980

MIII MARS 1981

OCTOBRE 1981

Figure 4

Evolution de la teneur du sol en Cd extractible par le DTPA pour les différents traitements boues.

Notes: Voir figure 2. 
M.S. ha ${ }^{-1}$; le fait qu'une fraction de l'épandage $190 \mathrm{t}$ M.S. ha ${ }^{-1}$ ait intéressé des boues non floculées (60 t M.S. ha ${ }^{-1}$ ) constitue un élément d'explication. Une fois de plus, les quantités extractibles déterminées après les nouveaux apports de boues sont très inférieures aux prévisions. Enfin, pour chacun des traitements, la concentration du sol en Cd extractible par DTPA paraît tendre vers une limite tout comme pour $\mathrm{Cu}$ et $\mathrm{Zn}$.

\section{Manganèse}

Nous avons inclus cet élément dans notre étude en raison de sa grande sensibilité à l'augmentation du pH du sol se traduisant par une réduction de son assimilabilité par les plantes. Les résultats obtenus en 1981 montrent des valeurs de Mn-DTPA significativement plus faibles, par rapport aux sols témoins, dans les sols ayant reçu des boues chaulées à une dose au moins égale à $70 \mathrm{t}$ M.S. ha ${ }^{-1}$. Les concentrations observées restent toutefois compatibles avec une alimentation correcte des végétaux.

\section{PLDTH-DTPA}

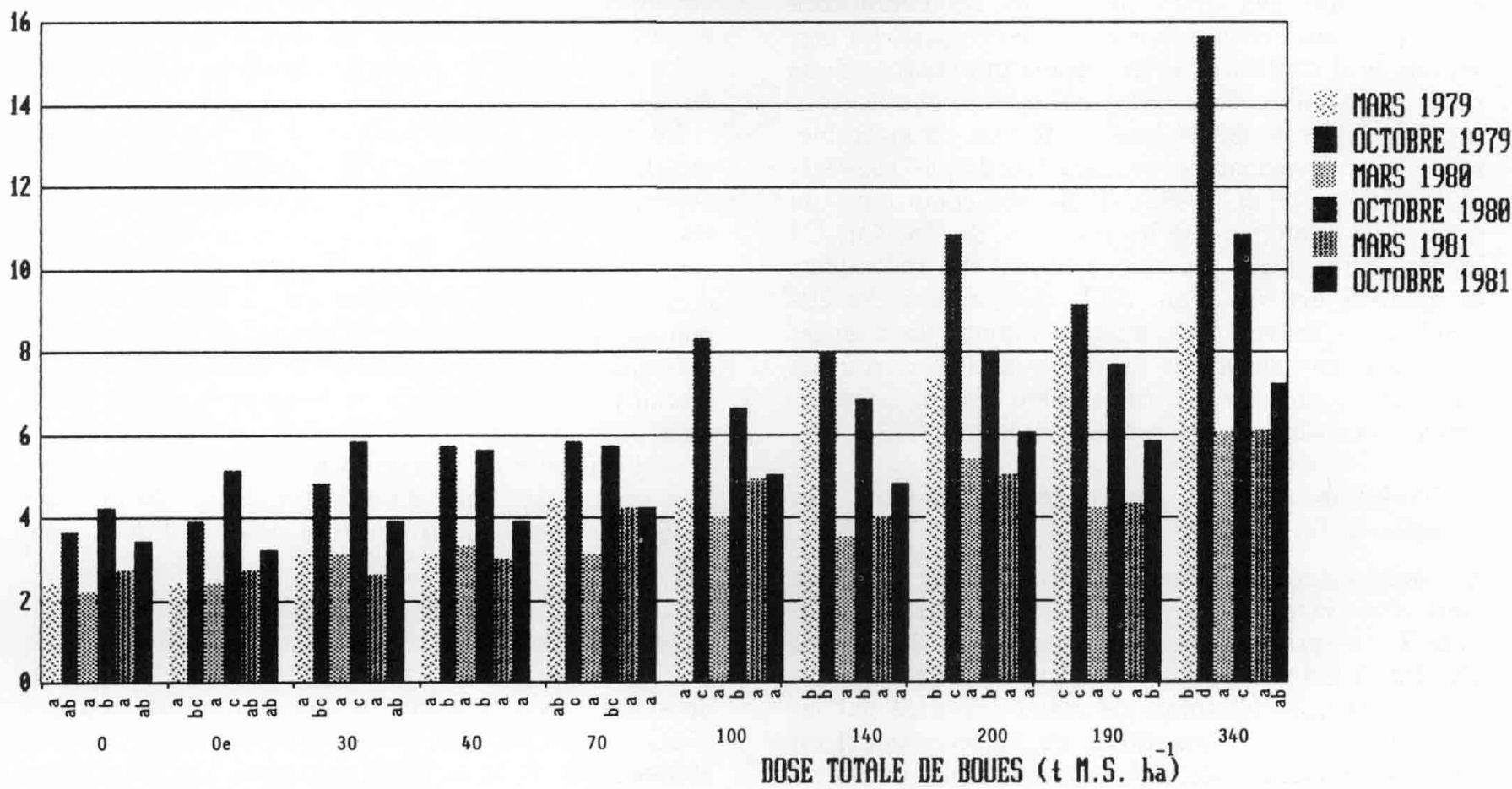

Figure 5

Evolution de la teneur du sol en Pb extractible au DTPA pour les différents traitements boues.

Notes : Voir figure 2.

\section{Plomb (fig. 5)}

L'évolution de Pb-DTPA est comparable à celle du cuivre. En mars 1979, la liaison entre Pb-DTPA et $\mathrm{Pb}$ apporté par les boues est très forte $(r=0,99)$. Avec une nette réduction de Pb-DTPA au cours du temps, on relève également d'importantes variations saisonnières pour tous les traitements y compris le témoin.

\section{Nickel}

Les données concernant cet élément montrent peu de variations en fonction des traitements et des saisons. Ce fait s'explique en partie par le taux d'extraction très faible obtenu pour le nickel avec le DTPA. La solution présente ainsi des concentrations souvent à la limite des possibilités de dosage.
Change in soil DTPA-extractable Pb for the different treatments. Notes: see figure 2.

\section{DISCUSSION}

\section{A. Evaluation de la méthode de prévision de l'assimi- labilité}

L'étude de l'évolution de la disponibilité de $\mathrm{Zn}$, $\mathrm{Cu}, \mathrm{Cd}, \mathrm{Pb}, \mathrm{Ni}$ et $\mathrm{Mn}$ pour les végétaux a été simulée, dans ce travail, à l'aide d'un extractant chimique complexant, le DTPA. La validité d'une telle démarche est déterminée par le niveau des corrélations entre le métal extractible par DTPA et sa concentration dans les tissus végétaux. Les corrélations sont généralement meilleures lorsque l'on introduit, dans les fonctions de prévision de l'absorption des métaux par les cultures, d'autres paramètres tels que $\mathrm{pH}$, taux de matière organique, CEC, taux d'argile (HAQ et al., 1980), mais on peut, dans un travail de type comparatif, se contenter en première analyse des résultats bruts « élements extractibles au DTPA ». L'utilisation des équations de prévision publiées par HAQ et 
al. (1980) montre en effet que les variations de $\mathrm{pH}$ et de matière organique ne sont pas de nature à modifier notablement les différences entre les quantités de métal assimilables. D'après les niveaux des corrélations signalées dans la littérature, on note que le DTPA rend des services très inégaux selon les éléments envisagés et selon les conditions de milieu. Il est tout à fait satisfaisant pour $\mathrm{Zn}, \mathrm{Cu}$ et $\mathrm{Mn}$, éléments pour lesquels il a été initialement développé dans le cadre des détections des carences en sols calcaires. Son intérêt a également été montré en milieu à forte teneur en métaux lourds pour $\mathrm{Zn}, \mathrm{Cu}, \mathrm{Cd}$ et $\mathrm{Ni}$ (KORCAK \& FANNING, 1978 ; HAQ et al., 1980). En sol alcalin cependant, les corrélations obtenues avec Ni sont médiocres (KORCAK \& FANNING, 1978), cet élément étant peu extractible, dans ces conditions, ainsi que nous l'avons constaté. D'autre part, les prévisions sont meilleures si les métaux ont été introduits dans le sol sous forme de sels que lorsqu'ils sont associés à une boue, les boues affectant considérablement le prélèvement des métaux lourds par les végétaux (GOMEZ et al., 1982). Dans nos conditions, on peut donc admettre que les quantités de $\mathrm{Zn}, \mathrm{Cu}, \mathrm{Cd}$ et Mn extraites par DTPA constituent des indicateurs acceptables des variations de la disponibilité des éléments pour les végétaux. Pour le plomb, les données manquent en raison des faibles quantités retrouvées dans les organes aériens, cet élément restant essentiellement localisé dans les racines (HEVESY, 1923).

\section{B. Evolution dans le temps de la solubilité des métaux dans DTPA}

L'action des boues sur les concentrations du sol en métaux extractibles par DTPA est encore très marquée 3 ans après le dernier épandage (mars 1979). $\mathrm{Zn}$, $\mathrm{Cu}, \mathrm{Pb}$ et $\mathrm{Cd}$ présentent des valeurs étroitement corrélées à la quantité totale de métal apportée par les boues $(r>0,95)$. L'amplitude de l'effet résiduel est de toute évidence liée à la concentration du métal dans la boue $(\mathrm{Zn}>\mathrm{Pb}>\mathrm{Cu}>\mathrm{Cd})$. A plus long

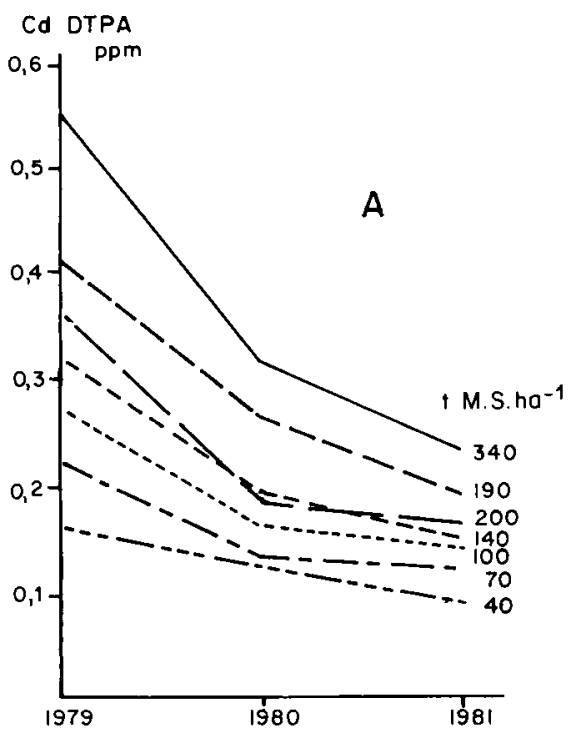

Figure 6

Evolution de la teneur du sol en Cd extractible par le DTPA : valeurs correspondant aux sols prélevés en octobre.

$A$ coordonnées décimales; $B$ coordonnées logarithmiques.

Note : les traitements 0 et 30 t M.S. ha ${ }^{-1}$ ne sont pas représentés. terme, 6 ans sans apports, l'influence des boues s'amenuise, mais la liaison concentration-dose reste très forte. Un nouvel épandage de boues (1979) entraîne une augmentation des quantités de $\mathrm{Zn}, \mathrm{Cu}$, $\mathrm{Pb}$ et $\mathrm{Cd}$ extractibles, l'accroissement cependant est bien inférieur aux valeurs prévisibles en tenant compte des effets des premiers épandages de boues. Cette observation, signalée par ailleurs à l'occasion d'apports répétitifs de boues (TAYLOR et al., 1982), réfute l'hypothèse d'une additivité des effets des boues. Il semble préférable d'admettre qu'il existerait, pour un type de sol donné, une limite de concentration en métal facilement mobilisable, laquelle pourrait dépendre à la fois des caractéristiques du sol et de la boue incorporée. Dans notre étude, le chaulage des boues contribue probablement à réduire la teneur du sol en métaux assimilables, particulièrement pour $\mathrm{Cd}$ et $\mathrm{Zn}$ dont la solubilité est étroitement liée au pH du sol.

Le suivi de l'évolution, au cours du temps, des métaux extractibles par DTPA paraît mettre en évidence, pour $\mathrm{Zn}, \mathrm{Cu}, \mathrm{Pb}$ et $\mathrm{Cd}, 2$ processus antagonistes :

- une évolution du métal vers des formes de moins en moins mobilisables qui, à l'image du cadmium (fig. 6), semble suivre une loi de type fonction puissance du temps et tendre vers une limite dont le niveau dépend de la dose de boue initialement appliquée ;

- l'existence de variations saisonnières se manifestant ici sous la forme d'un accroissement de l'extractibilité des métaux entre mars et octobre d'une même année.

Le premier processus, qui tendrait à limiter la contamination des eaux et des plantes par les métaux lourds, correspond à la fixation du métal observée, notamment à l'occasion de corrections de carences (FOLLET \& LiNDSAY, 1971), qui se traduit par un abaissement de la mobilité du métal. Ce phénomène ne peut être généralisé à tous les types de boues eu

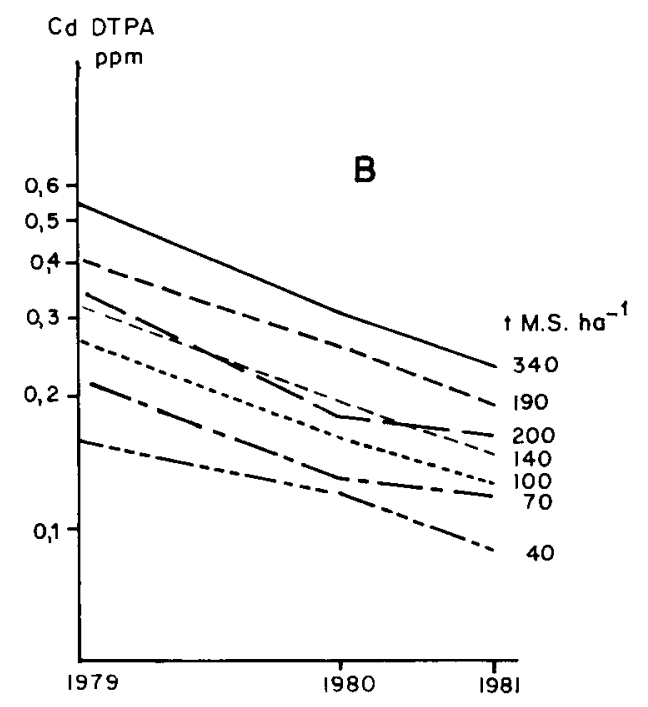

Change in soil DTPA-extractable Cd: October values. A decimal coordinates; $B$ logarithmic coordinates.

Note : treatments 0 and 30 tD.M. ha ${ }^{-1}$ are not represented. 
égard au mode de conditionnement particulier de nos boues. Il serait la résultante de l'intervention de plusieurs facteurs du sol (JUSTE \& SOLDA, 1979) dont il est impossible ici de cerner avec précision l'influence. La décroissance de la solubilité pourrait être, en premier lieu, attribuée à la diminution du stock de matière organique du mélange sol-boues (MOREL \& GUCKERT, 1981). En affectant les complexes organométalliques solubles, la minéralisation provoquerait le retrait des solutions du sol des métaux associés qui précipiteraient, s'adsorberaient ou s'incluraient dans les phases minérales du sol. Par référence au pouvoir complexant des composés fulviques extraits de sols enrichis en boues (SPOSITO et al., 1981), les éléments surtout concernés par ce mécanisme seraient $: \mathrm{Pb}, \mathrm{Cu}$ et, dans une moindre mesure, $\mathrm{Zn}$. Parallèlement, l'humification de la matière organique peut conduire à l'inclusion d'éléments métalliques dans des composés de masse moléculaire élevée, biologiquement de plus en plus stables. La stabilité de ces composés est accentuée par les liaisons qu'ils contractent avec les argiles et renforcée ici par l'effet de chaulage des boues. Un troisième type de processus propre aux boues chaulées peut être avancé pour expliquer la réduction de mobilité de $\mathrm{Cd}, \mathrm{Pb}$ et $\mathrm{Zn}$. Il s'agit de l'insolubilisation chimique soit par simple précipitation à $\mathrm{pH}$ élevé, soit par adsorption sur les surfaces du carbonate de calcium (Mac BRIDE, 1980). Enfin, le lessivage, responsable dans le cas de l'épandage de boues chaulées de l'apparition de $\mathrm{Cu}$ dans les eaux de drainage (JUSTE et al., 1977), peut constituer un facteur d'explication supplémentaire de la diminution de l'extraction de cet élément.

Les variations dites saisonnières pourraient résulter essentiellement de l'activité biologique du système solplante. Notre travail lève ainsi l'apparente contradiction entre les données de la littérature rapportant, selon les cas, une réduction ou un accroissement de l'extractibilité des métaux. Les processus de biodégradation, en provoquant une libération de complexes métalliques solubles $(\mathrm{Cu}$ et $\mathrm{Pb})$ et une acidification, sont susceptibles d'assurer la dissolution des composés carbonatés et la libération de cations initialement emprisonnés $(\mathrm{Cd}, \mathrm{Pb}, \mathrm{Zn})$. L'ampleur des remontées observées en octobre se réduit au cours du temps par suite d'un épuisement probable des matières organiques labiles et de la stabilisation des composés carbonatés. Les variations saisonnières sont ainsi moins marquées 2 ans après l'application des boues. Nous pouvons admettre également que le système racinaire de la culture installée peut modifier, par l'émission de substances complexantes et/ou acides dans la rhizosphère, l'état de solubilité des cations (MOREL et al., 1983). L'abondance de racines observées sur les parcelles à forte dose de boues confère à ce processus un rôle non négligeable. Il est évident que ces 2 types de phénomènes (biodégradation, activité du système racinaire) interviennent à des degrés divers selon les cations, en relation avec leur mode de rétention prédominant dans le sol. C'est ainsi que $\mathrm{Pb}$ et $\mathrm{Cu}$, très complexables par la matière organique, offrent des variations saisonnières très marquées, à l'inverse de
$\mathrm{Cd}$ et $\mathrm{Zn}$ dont l'état dans le sol dépend étroitement $\mathrm{du} \mathrm{pH}$ et de la présence abondante de $\mathrm{CaCO}_{3}$. Enfin, si la diminution de la mobilité est un phénomène permanent, il est masqué au cours de la saison de végétation par l'intervention de l'ensemble des processus qui conduisent à un accroissement de la mobilité des métaux.

\section{CONCLUSION}

Ce travail confirme l'augmentation de la teneur du sol en métaux lourds $(\mathrm{Zn}, \mathrm{Cu}, \mathrm{Cd}$ et $\mathrm{Pb}$ ) par suite de l'emploi de boues urbaines en agriculture. La fraction présumée assimilable de ces métaux dans le sol, déterminée à l'aide de DTPA, est apparue étroitement liée à la quantité totale de boues épandues. Au cours du temps, l'extractibilité des métaux par le DTPA a tendance à diminuer. A l'occasion d'un nouvel épandage de boues, on note que la quantité de métaux extraits s'accroît à nouveau mais reste à un niveau inférieur à la prévision, suggérant la non-additivité des effets d'apports successifs de boues. La perte de mobilité des métaux lourds au cours du temps pourrait être liée à un ensemble de processus tels que l'évolution des matières organiques par biodégradation (minéralisation et humification) et l'insolubilisation par précipitation ou adsorption. Cependant, selon les propriétés de l'élément, particulièrement selon son affinité pour les matières organiques, chacun de ces processus interviendrait à un degré variable.

$\mathrm{Au}$ cours de la saison de végétation, il semble que l'action de la microflore, d'une part, et du système racinaire, d'autre part, augmentent l'extractibilité par DTPA des métaux lourds dans le sol, ce qui se traduit par des variations saisonnières. La production de composés acides et/ou complexants s'oppose ainsi provisoirement à la fixation des métaux sous des formes moins mobiles. Toutefois, ces variations ont tendance à s'atténuer lorsqu'on s'éloigne de la date du dernier épandage. Ces phénomènes de mise en solution par intervention de l'activité biologique, qui restent à préciser au niveau des cinétiques notamment, revêtent sans doute une importance considérable dans le cas de zones à forte teneur, à la fois en métaux lourds et en matière organique.

Il est cependant difficile de dissocier au champ les différents processus impliqués dans les variations de mobilité des métaux pour quantifier la part revenant à chacun. Aussi, paraît-il indispensable de reprendre une étude plus fine de ces phénomènes à l'aide de modèles simplifiés. Des investigations en cours concernent en particulier le rôle des exsudats racinaires sur la mobilité des métaux lourds apportés au sol.

Reçu le 31 mai 1983. Accepté le 21 décembre 1983.

\section{REMERCIEMENTS}

Ce travail a fait l'objet d'une aide financière du Ministère de l'Environnement dans le cadre de la Convention $n^{\circ} 80180$. 


\section{RÉFÉRENCES BIBLIOGRAPHIQUES}

Chang F. H., Broadbent F. E., 1982. Influence of trace metals on some soil nitrogen transformations. J. Environ. Qual., 11, 1-4.

Chaussod R., 1979. Comment déterminer l'azote minéralisable des boues résiduaires, p. 253-260. In « First European Symposium treatment and use of sewage sludge », D. Alexandre \& H. Ott ed., Ministère de l'Environnement, France, C.E.C. Brussels, 479 p.

Emmerich W. E., Lund L. J., Page A. L., Chang A. C., 1982. Solid phase forms of heavy metals in sewage sludge-treated soils. J. Environ. Qual., 11, 178-181.

Fardeau J. C., Guiraud G., Hetier J. M., 1977. Répercussion de l'épandage de boues résiduaires sur la mobilité de $\mathrm{Cd}, \mathrm{Cr}, \mathrm{Hg}, \mathrm{Zn}$

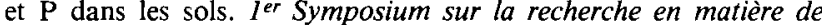
sols et déchets soildes. Orléans. Collection Recherche-Environnement $\mathrm{n}^{\circ} 11$.

Follet R. H., Linclsay W. L., 1971. Changes in DTPA-extractable zinc, iron, manganese and copper in soils following fertilization. Soil Sci. Soc. Am. Proc., 35, 600-602.

Godin P., 1982. Sources de contamination et enjeux, p. 3-23. Séminaire. Eléments traces et pollution des sols. Ministère de l'Environnement, France, $58 \mathrm{p}$.

Gomez A., Juste C., Nicibar B., Lessart P., Heres A., Alexandre D., 1982. Définition des seuils de phytotoxicité pour différents métaux susceptibles d'être rencontrés dans les boues de stations d'épuration pour des sols à très faible ou à très fort pouvoir de fixation vis-à-vis des cations. C.R. contrat d'étude $\mathrm{n}^{\circ}$ 79-81, Ministère de l'Environnement, $108 \mathrm{p}$. + annexes.

Guckert A., Kientzler L., Morel J. L., 1982. Etude de l'action à long terme de boues résiduaires urbaines chaulées riches en métaux lourds sur les propriétés d'un sol à fort complexe absorbant et sur les productions vézétales. C.R. contrat d'étude $\mathrm{n}^{\circ}$ 78-131, Ministère de l'Environnement, $66 \mathrm{p}$.

Haq A. U., Bates T. E., Soon Y. K., 1980. Comparison of extractants for plant-available zinc, cadmium, nickel and copper in contaminated soils. Soil Sci. Soc. Am. J., 44, 772-777.

Hevesy G., 1923. The absorption and translocation of lead by plants. Biochem. J., 17, 439-449.

Juste C., 1983. Problèmes méthodologiques: évaluation de la mobilité dans le milieu et de la disponibilité pour les plantes des éléments traces du sol et de certains déchets organiques. Bull. A.F.E.S. (à paraître)

Juste C., Lineres M., Gomez A., 1977. Etude du pouvoir complexant des métaux contenus dans les boues de stations d'épuration vis-à-vis des oligo-éléments et des éléments toxiques et action de ces complexes sur les végétaux. C.R. contrat d'étude $n^{\circ} 75-23$, Ministère de l'Environnement, $75 \mathrm{p}$.

Juste C., Solda P., 1979. Effets d'applications massives de boues urbaines riches en cadmium et en nickel sur une monoculture intensive de maîs, p. 372-382. In « First European Symposium treatment and use of sewage sludge " D. Alexandre \& H. Ott ed., Ministère de l'Environnement, France, C.E.C., 479 p

Kelling K. A., Keeney D. R., Walsh L. M., Ryan J. A., 1977. A field study of the agricultural use of sewage sludge. III. Effect on uptake and extractability of sludge-borne metals. J. Environ. Qual., 6, 352-358.
Korcak R. K., Fanning S. D., 1978. Extractability of cadmium, copper, nickel and zinc by double acid versus DTPA and plant content at excessive soil levels. J. Environ. Qual., 7, 506-512.

Latterell J. J., Dowdy R. H., Larson W. E., 1978. Correlation of extractable metals and metal uptake of snap beans grown on soil amended with sewage sludge. J. Environ. Qual., 7, 435-440.

Lindsay W. L., Norvell W. A., 1978. Development of a DTPA soil test for zinc, iron, manganese and copper. Soil Sci. Soc. Am. J., 42, 421-428.

Lineres M., Juste C., 1980. Mise au point de tests agronomiques permettant d'apprécier simultanément la phytotoxicité globale des boues de station d'épuration et la disponibilité pour les plantes des métaux qu'elles contiennent. C.R. contrat d'étude $n^{\circ}$ 77-145, Ministère de l'Environnement, $25 \mathrm{p}$.

Mac Bride M. B., 1980. Chemisorption of $\mathrm{Cd}$ on calcite surfaces. Soil Sci. Soc. Am. J., 44, 26-28.

Morel J. L., 1980. Effects of sludge processing on soil phosphorus mobility measured with the isotopic dilution kinetics method, p. 163-175. In « Phosphorus in sewage sludge and animal waste slurries " T. W. G. Huckert \& G. Gatroux ed., Reidel publishing company, Dordrecht Holland, $443 \mathrm{p}$.

Morel J. L., Guckert A., Sibout V., Jacquin F., 1978. Possibilités de valorisation agricole de boues résiduaires urbaines. I. Etude des variations de la composition des boues. Bull. E.N.S.A.I.A. Nancy, 20, 21-29.

Morel J. L., Guckert A., 1981. Influence of limed sludge on soil organic matter and soil physical properties, p. 25-42. In « The influence of sewage sludge publication on physical and biological properties of soils " G. Catroux, P. L'Hermite \& E. Suess ed., Reidel publishing company, Dordrecht Holland, $253 \mathrm{p}$.

Morel J. L., Guckert A., Chavanon M., Mench M., 1983. Etude des interactions entre les produits d'exsudation racinaire et les métaux lourds. I. Recherche d'une méthode de mesure de la capacité de liaison métallique des exsudats. Acta Oecol., Oecol. Plant., 4, 363-376.

Robertson W. K., Lutrick M. C., Yuan T. L., 1982. Heavy applications of liquid-digested sludge on three ultisols. I. Effects on soil chemistry. J. Environ. Qual., 11, 278-282.

Silviera D. J., Sommers L. E., 1977. Extractability of copper, zinc, cadmium, and lead in soils incubated with sewage sludge. $J$. Environ. Qual., 6, 47-52.

Sposito G., Holztclaw K. M., Levesque Madore C. S., 1981. Trace metal complexation by fulvic acid extracted from sewage sludge. I. Determination of stability constants and linear correlation analysis. Soil Sci. Soc. Am. J., 45, 465-468.

Sposito G., Lund L. J., Chang A. C., 1982. Trace metal chemistry in arid-zone field soils amended with sewage sludge. I. Fractionation of $\mathrm{Ni}, \mathrm{Cu}, \mathrm{Zn}, \mathrm{Cd}$, and $\mathrm{Pb}$ in solid phases. Soil Sci. Soc. Am. J., 46, 260-264.

Taylor R. W., Duseja D. R., Thangudu P. R., 1983. Heavy metal accumulation and movement in a sewage sludge amended soil. $J$. Environ. Qual. (à paraître). 